\title{
Décadrages Décadrages
}

cınéma, à travers champs Cinéma, à travers champs

12 | 2008

Fredi M. Murer

\section{Le paysage dans Höhenfeuer}

\section{Jacob Lachat}

\section{OpenEdition}

\section{Journals}

Édition électronique

URL : http://journals.openedition.org/decadrages/368

DOI : $10.4000 /$ decadrages. 368

ISSN : 2297-5977

\section{Éditeur}

Association Décadrages

\section{Édition imprimée}

Date de publication : 10 avril 2008

Pagination : 40-53

ISBN : 978-2-9700582-7-4

ISSN : 2235-7823

Référence électronique

Jacob Lachat, «Le paysage dans Höhenfeuer », Décadrages [En ligne], 12 | 2008, mis en ligne le 05 février 2014, consulté le 21 avril 2019. URL : http://journals.openedition.org/decadrages/368 ; DOI : $10.4000 /$ decadrages.368 
1 Cet article est le prolongement d'un travail écrit dans le cadre du cours-séminaire donné par Maria Tortajada au semestre d'automne 2007 à la Section d'Histoire et esthétique du cinéma de l'Université de Lausanne et intitulé: "Cinéma suisse: autour de Daniel Schmid, Fredi M. Murer et Thomas Koerfer".
2 "[La] vogue des 'Heimatfilme, était à son apogée dans les années cinquante, assurant en quelque sorte la pérennité du cinéma de divertissement et prolongeant la production du régime national-socialiste. C'était un cinéma fondamentalement apolitique, mais en évacuant le politique il masquait la réalité sociale et créait un monde de rêve, d'évasion et de distraction. Les ، Heimatfilme, [...] procuraient l'évasion au sein d'un univers innocent et préservé - sorte de cocon emblématique qui restait hors du monde réel même quand il était localisable [...].", Martin Schlappner, Martin Schaub, Cinéma suisse: regards critiques, 1896-1987, éd. Centre suisse du cinéma, Zurich, 1987, p. 38.

3 Martin Schaub, L'usage de la liberté: le nouveau cinéma suisse, 1964-1984, L'Age d'homme/Pro helvetia, Lausanne/Zurich, 1985, p. 135.
Le paysage dans Höhenfeuer ${ }^{1}$

par Jacob Lachat

A regarder les films de Fredi M. Murer, on prend conscience d'une parenté entre la plupart de ses œuvres, qu'elles soient de fiction ou documentaires: les univers diégétiques sont rarement localisables avec précision. Dans Wir Bergler in den Bergen sind eigentlich nicht schuld, dass wir da sind (1974), mis à part le dialecte dans lequel ils s'expriment, aucun indice ne permet de rattacher les paysans à un canton précis. De semblable manière, la périphérie urbaine de Grauzone (1979) pourrait correspondre à celle de n'importe quelle ville européenne. L'intérêt du cinéaste n'est donc pas de représenter un lieu déterminé mais un type de paysage (ville, désert, campagne, etc.).

Dans Höhenfeuer (1985), Murer semble, une fois de plus, empêcher toute identification géographique précise au profit d'un "pur» cadre alpestre. Or, en réalisant une fiction dans un tel cadre, le réalisateur s'aventure dans un univers qui n'est pas sans évoquer celui du Bergfilm ou du Heimatfilm. En effet, pour les «nouveaux» cinéastes helvétiques des années 1960-1970, la montagne est communément associée à ces deux genres filmiques dont les principaux titres se situent entre les années 1930 et 1960. Et dès les années 1960, ces jeunes réalisateurs de gauche ont vivement fustigé ces genres, arguant non seulement qu'ils étaient sous-tendus par une idéologie traditionaliste, mais également qu'ils proposaient une image archaïque et déformée de la Suisse contemporaine ${ }^{2}$. La montagne y occupe une place centrale: d'une part, elle constitue un environnement au caractère suffisamment terrifiant et infranchissable pour élever ceux qui en entreprennent l'ascension au rang de héros; d'autre part, elle est le lieu privilégié des clichés paysagers. Elle se résume en effet à quelques traits caricaturaux:

«[...] de verts pâturages où paissent quelques vaches; un lac bleu, tranquille comme un miroir; des forêts vert sombre un peu plus haut; plus haut encore, toute une palette de tons gris, dominée par des sommets d'une blancheur immaculée: la neige, dite ‘éternelle> dans le langage mythique; un ciel bleu, tout proche, coiffe le tout comme un toit protecteur.» $\mathbf{3}$ 
Ainsi considérées, de telles images ne peuvent guère ne pas paraître surannées au moment où Murer réalise son film. Or il est déconcertant de constater que tous ces clichés - hormis peut-être celui du lac bleu - figurent précisément dans Höhenfeuer. A bien des égards, les images semblent renouer avec le culte de la belle montagne. Pourtant, la représentation du paysage n'atteint jamais une dimension stéréotypée. On peut donc postuler un décalage par rapport au fameux «mythe alpestre " $\mathbf{4}$, qui invaliderait une association entre cette production et les Heimatfilme. Le spectateur en est d'autant plus surpris que le film ne paraît pas non plus se situer dans le sillage des productions qui ont contribué à déconstruire cette peinture esthétisante de la Suisse. Murer se révèle en effet éloigné d'un Tanner, dont les films sont souvent porteurs d'un discours critique envers l'imaginaire national 5 . Peut-on donc affirmer que Höhenfeuer s'inscrit en marge des débats idéologiques sur le paysage identitaire helvétique? Plus précisément, il semble que l'un des enjeux principaux du film de Murer consiste à redonner à la montagne une symbolique forte, en évitant tout à la fois l'exaltation que prônait la «Défense nationale spirituelle» 6 et la critique caustique (parfois très rigide) soutenue par le "nouveau» cinéma.

Pour interroger cette «nouvelle» symbolique, j’ai décidé d'aborder le film sous un angle prioritairement esthétique. Quelle montagne représente-t-on? qui la regarde? quand et comment? sont autant de questions qui portent sur la nature et la fonction du paysage au sein de Höhenfener. Je tenterai d'éclaircir ces différents points, non par une étude de longues séquences autonomes, mais par une analyse précise de certains brefs moments du film. En effet, bien qu'elle soit essentielle au déroulement narratif du récit, la montagne n’y est représentée que sporadiquement. Je commenterai donc ici quelques micro-séquences, voire quelques plans, dans lesquels celle-ci semble investie d'une véritable signification.

\section{Sortir du Heimatfilm}

Höhenfeuer est souvent décrit comme un drame psychologique sur l'inceste - Murer parle même de tragédie grecque $\mathbf{7}$. Ancré dans une montagne retirée, ce récit traite d'un amour fraternel entre deux enfants qui se transforme en amour charnel. Univers alpestre et amours enfantines: ces deux éléments narratifs pourraient a priori faire penser que le film s'inspire de Heidi und Peter (Schnyder, 1954). Ils pourraient également être assimilés aux sujets des petites tragédies familiales du cinéma suisse des années 1950. Ces rapprochements sont toutefois peu pertinents, d'une part parce que le paysage, dans le film, n'est pas représenté comme un cadre féerique, conventionnellement beau et propice à l'éclosion
4 Claude Reichler propose un historique de l'origine de ce mythe alpestre dans La découverte des Alpes et la question du paysage, Georg, Chêne-Bourg, 2002, p. 10.

5 Je pense notamment à Messidor (1979). Dans ce film, les deux protagonistes voyagent à travers la Suisse à la manière d'un jeu, qu'elles considèrent comme celui "du temps et de l'espace vides". Ces deux qualificatifs, qui caractérisent le paysage helvétique, sous-entendent bien la visée critique qu'adopte le réalisateur.

6 II s'agit de la culture morale instaurée par le gouvernement suisse entre les années 1930 et 1950 , et destinée à favoriser une certaine unité nationale face à la propagande des pays alors en guerre. Dans ce contexte, les Alpes, l'espace rural et les valeurs qui lui sont conférées deviennent autant de symboles de l'identité suisse. A ce sujet, voir notamment Hervé Dumont, Histoire du cinéma suisse: Films de fiction 1896-1965, Cinémathèque suisse, Lausanne, 1987, pp. 236-237.

7 Voir notamment: Fredi M. Murer, Höhenfeuer: ein Werkstattbuch, Baumann \& Stromer, Zurich, 1986. 


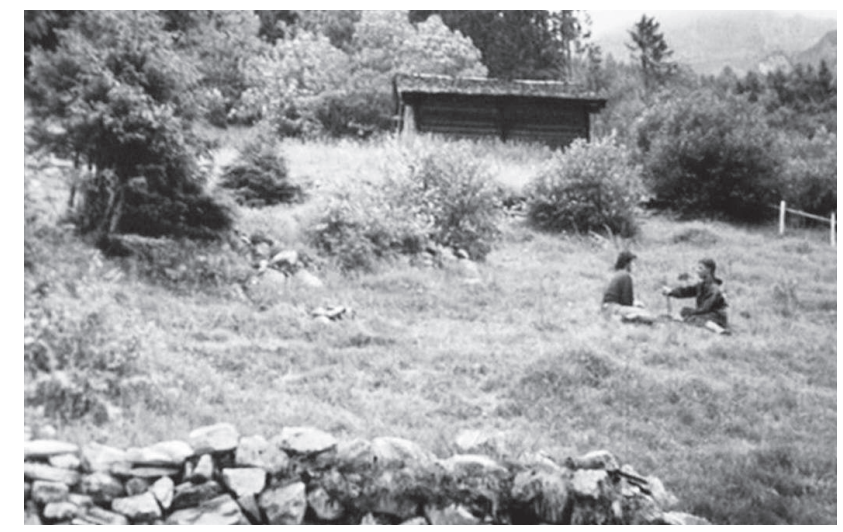

8 Fredi M. Murer, "De la montagne et du tournage en montagne", dans Walter Ruggle (éd.), Univers de Montagnes. L'année internationale de la montagne 2002. Cycle de films, TrigonFilm, Direction du développement et de la coopération, 2002, p. 17 [Brochure éditée à l'occasion d'un cycle de films de montagne ayant circulé dans différentes localités suisses entre juin 2002 et février 2003].

d'un amour; et d'autre part parce que la transgression que constitue l'inceste est particulièrement violente.

Comme dans tout film narratif, le paysage n'est à l'évidence pas choisi au hasard. Il est intimement lié au déroulement du récit et constitue pour lui un univers qui infléchit le destin des personnages. Or, à l'ouverture, Höhenfeuer est ancré dans un cadre géographique peu déterminé. Aucune mention ne précise dans quelle région se situe la ferme familiale, ni quelle est cette montagne qui surplombe la vallée. Cependant, ces deux éléments du paysage sont soulignés par un lent travelling latéral droit qui longe en contre-plongée le terrain pentu (fig. 1). Sur fond d'une musique nappée de sons étranges, on voit le Bouèbe (Thomas Nock) et sa sœur Belli (Johanna Lier) affairés à déterrer deux souris mortes. Derrière eux se dresse une cabane et, en arrière-fond, la montagne se distingue à peine. Soudainement, la caméra passe en gros plan sur les visages des enfants: un court champ/contre-champ les montre en train de contempler leurs proies et d'échanger des regards intermittents. Ces raccords abrupts ne constituent pas uniquement un effet stylistique mais permettent d'accentuer une lecture fictionnalisante.

«Pour ne pas risquer de faire [avec Höhenfeuer] un film de terroir, nous avons eu recours à un artifice qui nous a permis de transformer un paysage authentique en paysage de pure fiction. Nous avons systématiquement et radicalement éliminé les lignes d'horizon du champ visuel: toute identification géographique est ainsi devenu impossible, une région reconnaissable se transforme en idée de paysage alpin. » 8

Selon le cinéaste, un film qui permet d'identifier géographiquement un paysage impliquerait une représentation documentarisante - ou en tout cas aurait partie liée avec elle, puisqu'il l'oppose à la "pure fiction». Par 
cette déclaration Murer ne veut pas tant, semble-t-il, désigner le genre cinématographique auquel appartient cette histoire - le drame - que souligner à quel point celle-ci est loin d'une problématique régionaliste. Car on ne sort pas de Höhenfeuer comme d'un film sur la paysannerie suisse de montagne. Le cinéaste ne fait ni référence à une réalité "afilmique", ni à un contexte spatial repérable par le spectateur. Dans une telle optique, la musique extra-diégétique joue également un rôle signifiant. A l'instar de l'image au traitement "dépaysant», elle encourage une lecture fictionnalisante, et cela d'autant plus que son caractère sibyllin (qui l'éloigne des airs traditionnels suisses) confère au récit une dimension onirique et presque intemporelle.

Toutefois, bien que Höhenfeuer donne parfois l'impression de se dérouler dans une période indéterminée, son récit se révèle entièrement ancré dans l'époque contemporaine. En effet, tout au long du film, Murer ne cesse de mettre en évidence les signes d'une certaine modernité : la pompe motorisée, l'électricité, le téléphérique, la radio, etc. Cette profusion d'éléments technologiques indique que le retrait face au monde ne fait pas de la famille une charmante société autarcique. Au contraire, ses membres dépendent du village qu'ils côtoient indirectement. Lorsque le père descend en plaine et qu'il en rapporte des cadeaux, ses retours sont vécus comme de véritables respirations. Car, dans cette montagne et plus encore dans cette ferme aux pièces exiguës, c'est avant tout l'impression d'une claustration que traduisent les personnages. Belli, malgré le temps qu'elle consacre à ses lectures, éprouve une lassitude atroce à vivre loin du monde et de son ami, dont elle ne peut recevoir que les lettres. La mère, quant à elle, est victime de crises d'asthme, qu'elle subit (symboliquement ?) à sa fenêtre, devant un paysage silencieux et morne.

Ce paysage, c'est celui que le début du film donne à voir. Alors que le père arrose au purin le pâturage incliné, la caméra filme en plongée, l'instant d'un court plan fixe, le flanc d'une vallée embrumée (fig. 2). Dans la mesure où le bruit hors-champ du moteur de la pompe atteste le lien entre le paysage exposé et le lieu de l'action, ce plan, quelque peu abrupt, donne à voir l'étendue de cette vallée telle qu'elle apparaît aux personnages. Plutôt qu'une nature idyllique, Murer préfère montrer une nature maussade, imprégnée de grisaille. Elle apparaîtra même souillée par le geste du père: un geste qui signifie que le paysage qui s'offre au regard est aussi celui que l'on alimente d'engrais (fig. 3). De plus, l'ensemble de cette brève séquence manifeste un humour pour le moins trivial: sous les éclats de rire de sa fille, le père va même jusqu'à "puriner»son fils, qui tente autant que possible de protéger les fleurs du terrain. Face à de telles images, le spectateur se trouve loin de la nature
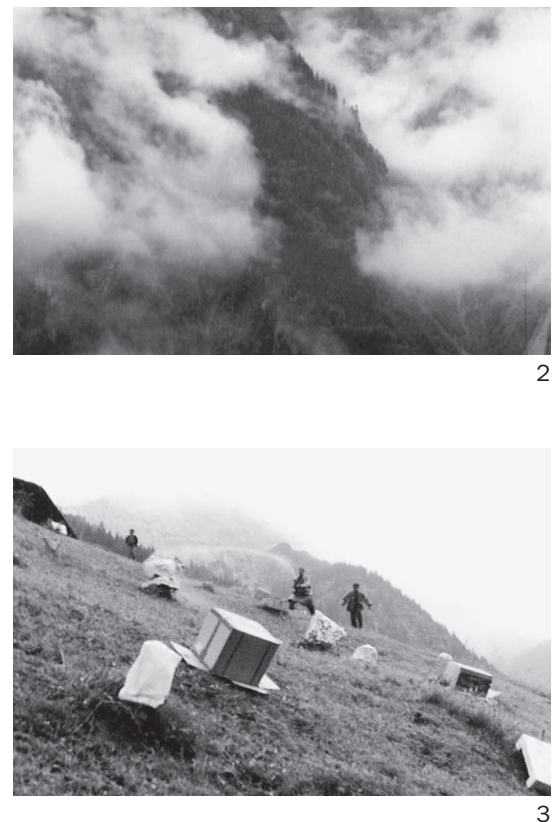
9 Maria Tortajada, "Cinéma suisse: comment échapper au paysage narcissique?", dans Derrière les images, Marc-Olivier Gonseth, Jacques Hainard, Roland Kaehr (éd.), Musée d'ethnographie, Neuchâtel, 1998, p. 281.

10 La fabrication du premier de ces amoncellements de pierre est d'un côté présentée comme une punition pour calmer la fougue de l'enfant. D'un autre, elle prend une signification plus traditionnelle: à chaque génération, lorsqu'un mâle "irascible" (dénomination attribuée aux membres de la famille pour leur colère atavique) devient adulte, il construit son propre mur. Une fois son ouvrage achevé, le Bouèbe, à l'instar de son père, se montre fier d'avoir marqué sa création, comme s'il en allait de son propre corps. Cependant, outre ce rituel "thérapeutique " et initiatique de surface, ces murs de soutènement ont surtout une fonction proprement matérielle: ils sont destinés à contenir la poussée du terrain incliné. immaculée du Heimatfilm. Cette scène inscrit au contraire le paysage filmé sous le signe du "désenchantement».

Ce paysage désenchanté, on sait ce qu'il signifie pour les "nouveaux" cinéastes suisses: c'est celui d'une pensée qui s'inscrit en porteà-faux contre l'idéologie fréquemment présente dans les Bergfilme puis dans les Heimatfilme, qui reposait pour l'essentiel sur des valeurs telles que la virilité, l'héroïsme et la maîtrise de soi. C'est aussi celui d'une représentation sans tromperie et sans emphase, une représentation vidée de tout l'imaginaire rousseauiste. En effet, les années 1960 et 1970 ont vu éclore une critique virulente à l'égard de la mentalité ambiante qui percevait dans le paysage alpestre un paradis perdu, un nouvel Eden, bref une nature dans laquelle l'homme, loin de la société "dissolue", retrouve la pureté originelle. Comme Maria Tortajada l'a expliqué, les "nouveaux" cinéastes ne se sont pas simplement interdit de filmer la montagne sous prétexte qu'elle était "inutilisable»; ils s'y sont au contraire intéressés de près en tâchant d'en déconstruire la dimension mythologique. Ainsi, ils l'ont d'une part "dépaysagée», en mettant à mal sa beauté conventionnelle et sa composante idéologique; d'autre part en la "faisant paysage", c'est-à-dire en proposant une redéfinition de sa nature esthétique 9 .

Une telle déconstruction/reconstruction est bien à l'œuvre dans Höhenfeuer. La scène du purin montre à quel point le film diverge de l'image aseptisée de la montagne que donnaient à voir les Heimatfilme. Si cette montagne paraît si peu étincelante, c'est que la nature n'est pas inaltérable. Le paysage alpestre est là, mais toujours parsemé d'empreintes que laisse l'homme, ainsi qu'en témoignent les murs construits par le Bouèbe10. Par là même, Murer déconstruit non seulement l'idée d'une montagne immuable, mais redéfinit aussi la "communion» entre l'homme et le paysage qu'il habite. En effet, si le lieu de l'action n'est pas identifiable, il ne peut pas non plus être associé à des valeurs morales reconnaissables, comme l'attache à la région. L'intérêt de Murer pour une paysannerie peu pittoresque débouche alors naturellement sur une symbolique différente du paysage: la montagne de Höhenfeuer devient ainsi pour le cinéaste un univers propice à la réalisation d'une fiction qui ne tombe pas dans les stéréotypes du Heimatfilm.

\section{La fonction narrative du paysage}

Le monde de Höhenfeuer ne renvoie donc pas à un lieu référentiel précis, même pour un spectateur helvétique. Il ne consiste pas non plus en un fond immuable de montagnes suisses qui ne sont d'ailleurs que rarement représentées. L'univers diégétique est marqué par une présence 
humaine: il est "pratiqué», transformé, sillonné; les personnages s'y déplacent et y communiquent autant que faire se peut. A première vue, ce monde construit un espace en tant qu'ensemble relationnel11.

Mais comment un tel univers est-il présenté ? Apparaît-il comme un ensemble homogène, ou au contraire, est-il hétéroclite? Et si tel est le cas, que devient la représentation du paysage dans un espace filmique divisé ? Si présent soit-il dans le film de Murer, l'espace ne l'est jamais de façon globale: il est soumis à un morcellement en deux lieux et en deux niveaux : les fermes familiales (celle des parents et celle des grandsparents) sont situées chacune sur un flanc de la vallée; et au-dessus de la ferme paternelle se trouve le refuge dans la montagne. Cette configuration simplifiée, qui permet de s'orienter dans l'univers filmique, permet de montrer comment la représentation du paysage résulte d'une organisation spécifique ${ }^{\mathbf{1 2}}$, divisible, elle aussi, en différents ensembles. Aussi, je propose d'opérer une distinction des multiples fonctions que revêt l'univers alpestre de Höhenfeuer en m'appuyant sur le modèle qu'envisage André Gardies dans une typologie du paysage 13. Ce modèle distingue les rôles qu'endosse celui-ci en six ensembles discursifs ${ }^{\mathbf{1 4}}$, parmi lesquels j'en retiens volontairement deux, le "paysage-fond» et le "paysagecatalyse», car ils permettent d'aborder le problème de la montagne en la considérant d'une part comme un décor et, d'autre part, comme un lieu qui influence directement les actions des personnages.

Les plans de paysage apparaissent souvent lors de transitions scéniques, c'est-à-dire qu'ils marquent le passage d'un temps de l'action à un autre. Le "paysage-fond» qui assure originellement l'ancrage spatial de la diégèse semble ainsi être avant tout un moyen de faire avancer la temporalité du film. A cet égard, le plan qui fait suite à la scène du mur est éloquent. Après avoir souligné par un gros plan la date («1984») de la construction du premier mur de pierre, érigé suite à la première "bêtise» du Bouèbe, la caméra filme en plan fixe un pré herbu que le vent effleure délicatement (fig. 4). Cet insert surgit comme une plage
11 "L'espace [est] composé d'un ensemble de traits particuliers, eux-mêmes saisis au sein d'un réseau relationnel [...].", André Gardies, L'espace au cinéma, Méridiens Klincksieck, Paris, 1993, p. 70.
12 A propos de cette organisation, j'ai remarqué que la présence du paysage est relativement plus rare dans la seconde partie du film. En effet, la fréquence de sa représentation ne cesse de croître jusqu'à ce que le Bouèbe atteigne le refuge. On constate à cet égard que la première moitié de la narration repose sur une ascension géographique: le lieu de l'action passe de la ferme au sommet de la montagne. Mais il est difficile d'expliquer le rapport exact entre cette ascension et la figuration décroissante de la nature.

13 André Gardies, "Le paysage comme moment narratif ", dans Jean Mottet (éd.), Les paysages du cinéma, Champ Vallon, Paris, 1999, pp. 141153.

14 André Gardies nomme ces six types de paysage cinématographique ainsi: "paysagefond "; "paysage-exposant"; "paysage-contrepoint"; "paysage-expression"; "paysage-catalyse"; "paysage-drame". (Ibid.) Bien que cette distinction soit, je crois, trop étanche, le registre esthétique que comporte chacune de ces catégories reste toutefois utile à l'objet de cette étude.

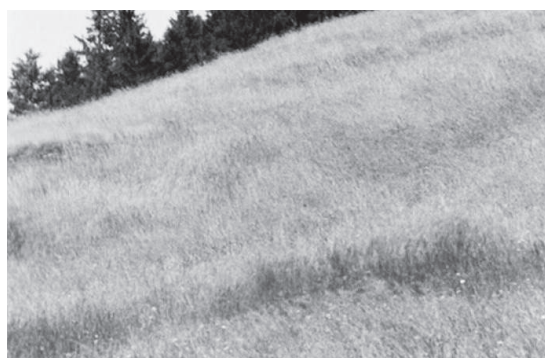


descriptive gratuite, créant un effet de surprise. Il donne à voir en contreplongée la bise se lever sur une pente dépourvue de présence humaine. Comme le plan sur la vallée nébuleuse décrit plus haut, ce pré apparaît sans qu'il soit motivé par un raccord regard. Annonce-t-il la destruction de la tondeuse, qui déclenchera le contentieux entre le père et son fils? Ou Murer tient-il surtout à souligner, par le vent, la fin de l'été? Dans le film, d'autres plans de paysage signalent d'ailleurs la succession des saisons: une vue en plongée de la maison au-dessus d'un "lac» de brume survient pour indiquer l'arrivée de l'hiver (fig. 5); plus tard, dans une autre plongée, la ferme apparaît sur le flanc de la montagne enneigée (fig. 6). Ces différentes occurrences sont assurément des descriptions de paysages, puisqu'elles n'évoquent aucune activité humaine. Grâce à elles, Murer décrit non seulement la scène du drame (la montagne), mais également la temporalité (les saisons) dans laquelle vivent les personnages. Par conséquent, le "paysage-fond» se double le plus souvent d'une autre fonction, que Gardies n'envisage pas dans sa catégorisation : le "paysage-temps».

En outre, Murer insiste sur une autre fonction du paysage via la représentation du rapport que le Bouèbe entretient avec la montagne. En effet, l'épisode de sa fuite dans le refuge fait de l'espace montagnard le lieu où va s'opérer la maturation du garçon. L'adolescent y construit non seulement, avec un acharnement démesuré, des murs de pierre,
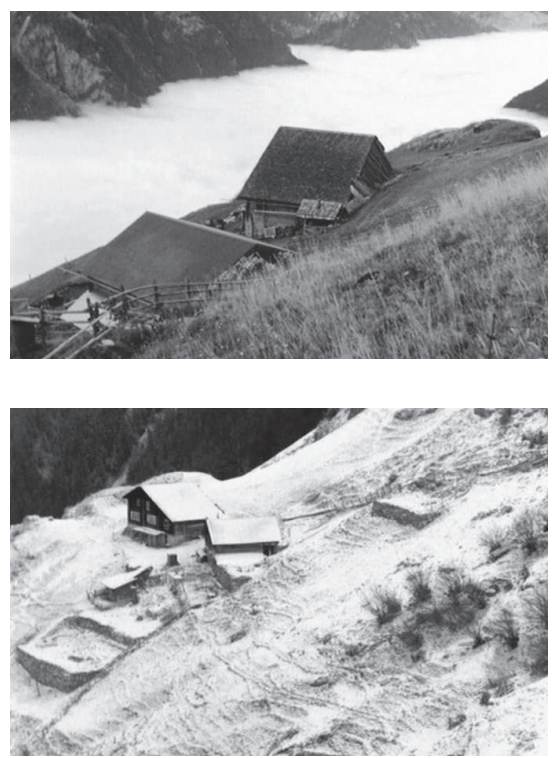


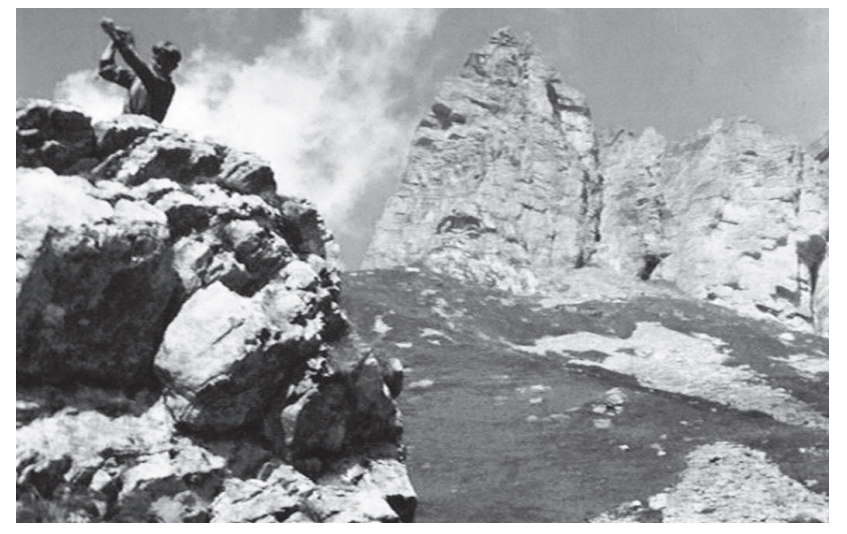

ainsi que son père le lui a enseigné, mais il y découvre surtout l'amour. Dans cette perspective, la montagne favorise le passage de l'enfant à l'homme, et elle constitue donc un véritable "paysage-catalyse», au sens de Gardies, puisqu'elle est « une composante de la diégèse susceptible de produire [...] la transformation d'une autre composante de cette même diégèse » 15 .

De plus, si ce cadre diffère autant de celui de la vallée, c'est que Murer le présente avant tout comme un endroit favorable à l'élévation (dans les deux sens du mot) du protagoniste. A peine est-il installé dans son refuge que le Bouèbe est immédiatement représenté avec la montagne en fond. Ce rapport intime entre le personnage et son nouveau paysage se manifeste par un lent panoramique en contre-plongée, grâce auquel la caméra glisse du sommet de la montagne au héros, que l'on voit occupé à extraire les schistes d'un rocher (fig. 7). Par un effet de perspective, la tête de l'enfant apparaît à la même hauteur que la cime, comme si le cinéaste voulait à la fois conjoindre et sublimer les deux "corps» dans le même plan. En ce sens, ce nouveau rapport entre personnage et paysage renverse le rapport que propose la représentation de la vallée qui, elle, instaure l'idée d'une distance entre les personnages (le fossé) 16.

Or, le lieu où le Bouèbe se réfugie est aussi irrégulier qu'exceptionnellement plat: le personnage y construit sa muraille en longueur et s'y meut à l'horizontale. Si bien que lorsque Belli le rejoint et lui manifeste sa présence, la caméra survole la surface l'instant d'un travelling latéral et suit le frère qui court à la rencontre de sa sœur (fig. 8). Contrairement à la ferme où tout est vertical, où les plongées et les contre-plongées présentent un territoire en pente aiguë qui semble annoncer à tout moment
15 ld., p. 149.

16 Elle suggère aussi l'idée d'une communication frontale entre la maison familiale et celle des grands-parents. 


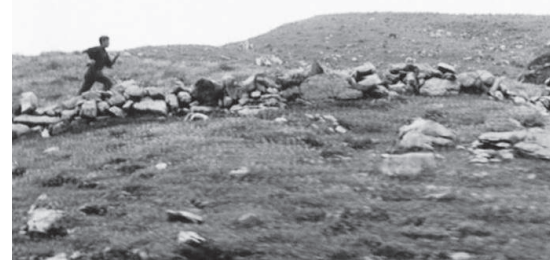

8
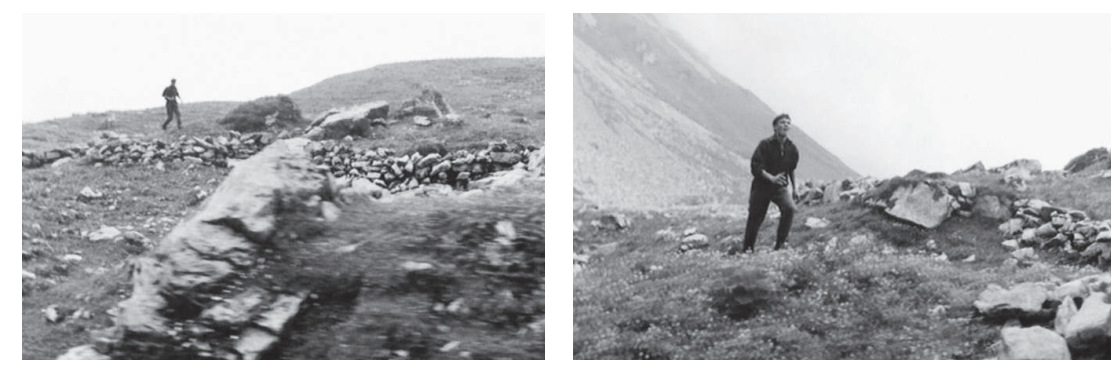

la chute des êtres (fig. 9), l'univers du refuge donne, quant à lui, l'impression à la fois d'être stable et de permettre une élévation.

C'est en effet dans cet Eden, au coin du «Feu des hauteurs» (Höhenfeuer), que les enfants expriment leur amour en silence et en parfaite innocence. Par ce geste, le sommet de la montagne devient un lieu où les codes familiaux et la morale sociale perdent leur sens, où les rapports humains ne sont plus réglés en fonction de principes normatifs propres aux lieux "d'en bas». Un rapport dialectique s'instaure ainsi entre l'univers de la famille (la plus petite forme de société) et celui des enfants : les valeurs prônées dans l'un n'ont pas cours dans l'autre. C'est pourquoi, dans ce contexte, la montagne se montre à l'image de l'élan de liberté qui anime le couple. Au contraire du Heimatfilm, qui représentait les Alpes comme un lieu de liberté essentiellement collective et identitaire (le peuple suisse), Murer choisit d'en faire un paysage pour une liberté individuelle et transgressive.

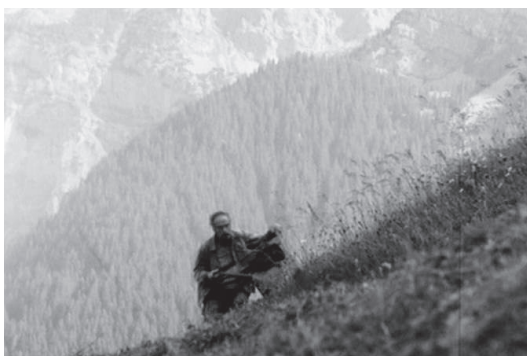

\section{Un point de vue multiple}

Le paysage de Höhenfeuer apparaît souvent à l'image tel qu'il est vu par le Bouèbe. Sous beaucoup d'aspects, le film semble même être en partie fondé sur sa perception visuelle. Dans les œuvres de fiction, il n'est pas rare que la représentation du monde diégétique traduise le point de vue 

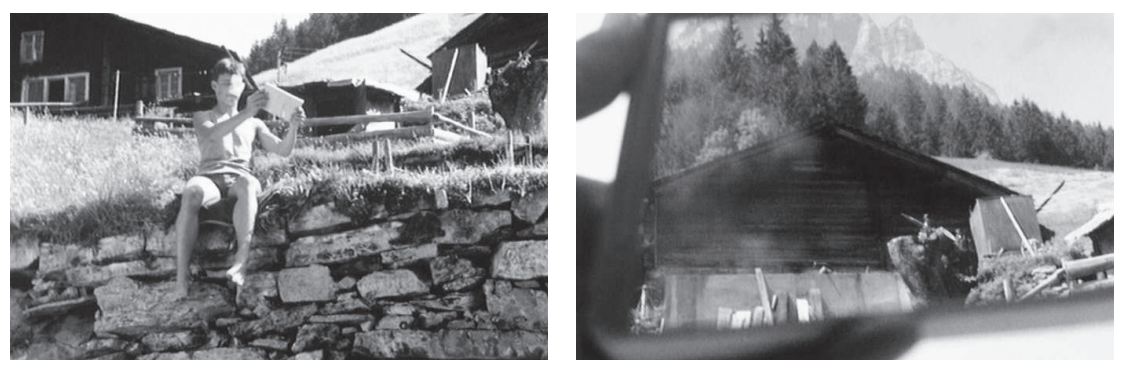

d'un ou plusieurs des protagonistes. Dans Höhenfeuer, cette représentation constitue à l'évidence l'un des enjeux principaux du film. Dès les premières minutes, le paysage s'avère effectivement être le fruit d'un travail rigoureux sur le point de vue : la ferme et la montagne apparaissent d'abord en contre-plongée lors de l'ouverture du récit et, plus tard, lorsque le Bouèbe les voit se refléter en arrière-plan dans son miroir (fig. 10). Son regard sur le paysage ainsi que celui de la caméra sont en effet ceux que Murer met en évidence, en passant de lents plans fixes ou en travelling à des mouvements d'appareil censés mimer ceux des yeux du personnage. Par ce biais, c'est non seulement l'idée d'un double regard sur le paysage qui est suggérée, mais également celle d'un dispositif qui fait converger l'univers vu par le protagoniste et celui vu par le «monstrateur»17. Comme le récit est organisé autour de l'évolution d'un enfant sourd qui est donc contraint de recourir à ses yeux pour déchiffrer ce qui l'entoure, la question du point de vue est, de fait, immédiatement rapportée à son regard. Dès lors, avec une telle focalisation (que la terminologie de Jost nomme "ocularisation interne primaire»18), la représentation du paysage participe principalement d'une volonté de souligner ce qui, dans le monde de la fiction, fait référence à une réalité subjective. Or, celui qui observe le paysage ne le contemple pas à distance, il vit pour ainsi dire dedans. Il ne le sublime pas non plus, comme certains ont tendance à magnifier ce qui leur paraît inatteignable ou trop précieux. Au contraire, il agit sur lui, avec lui. Il y a, dans le film, une interaction entre le Bouèbe et le paysage qu'il regarde. Avant de représenter un paysage, Murer montre un paysage "vu par», un paysage (littéralement) spectaculaire, qui n'a de sens que dans la mesure où il se constitue par rapport à une perception singulière.

Pour véritablement comprendre la fonction de cette perception si particulière de la montagne, il faut d'abord interroger l'attitude "voyeuriste» de celui qui la regarde. Voilà précisément le problème que pose la représentation en abyme. Le Bouèbe n'est pas absorbé par l'étendue

17 André Gaudreault distingue la monstration, l'acte de montrer, de la narration qui est au niveau second de l'organisation. Alors que la narration relève du montage, cette monstration relève, elle, du tournage; par conséquent, la représentation (du paysage, des personnages, etc.) dépend de l'instance nommée "monstrateur". Du littéraire au filmique: système du récit, Armand Colin, Paris, 1999.

18 Dans L'CEil-caméra, François Jost différencie ce qui a trait au savoir narratif du récit filmique (la focalisation proprement dite) du plan spécifiquement visuel (l'ocularisation). L'œil-caméra: entre film et roman, PUL, Lyon, 1989. 


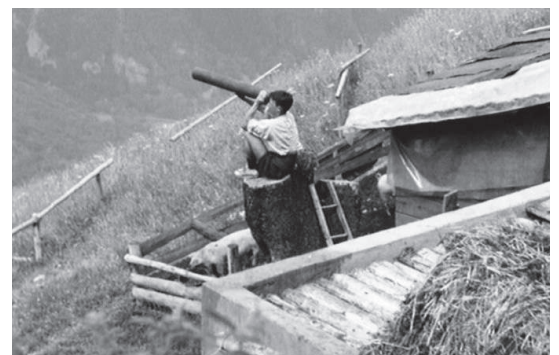

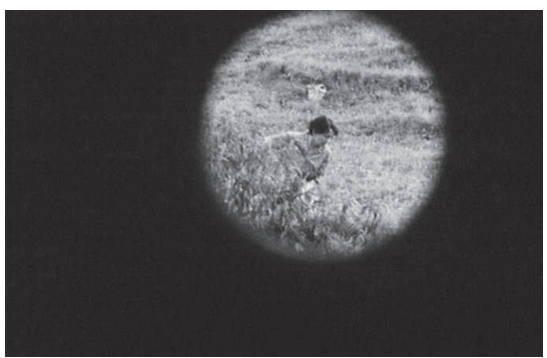

d'une nature ou d'une région, mais il ne cesse d'agrandir et de parcourir du regard les composantes de son univers. En ce sens, les objets d'optique auxquels il recourt sont d'une importance primordiale, puisqu'ils permettent, même de loin, d'attirer l'attention du spectateur sur les éléments accessoires du décor. Dans la scène où les enfants se rendent chez leurs grands-parents, le Bouèbe est captivé par la loupe, grâce à laquelle il examine attentivement les moindres détails physiques de son grand-père. Ici, la caméra suit ce point de vue qui, en passant des uns aux autres, agrandit les éléments auxquels il est rivé : d'abord la bouche, source de la parole; ensuite l'alliance, symbole du lien conjugal; enfin la montre, image canonique du temps qui passe. Autant de signes auxquels le personnage est étranger, dans la mesure où, au sein de sa surdité les objets prennent une signification quelque peu différente. Dans le film, cette façon d'isoler des gros plans, d'opérer un découpage presque analytique, se retrouve lorsque le Bouèbe scrute l'environnement de la ferme familiale au moyen d'un tube métallique et qu'il s'arrête sur différents éléments (fig. 11). Dans le cadre, le cercle de visibilité se meut avec hésitation, s'arrête sur un groupe de fleurs, puis quelques instants sur la mère. Il reprend ensuite son mouvement, remarque l'arrière-train d'une vache dans l'étable et finit par se figer sur Belli, que l'on voit danser en musique. Manifestement, ces différents «objets » sont autant d'éléments qui existent (au sens fort du terme) pour notre voyeur: il protège en effet les fleurs du purin; il joue avec les animaux dans la fange, comme s'ils étaient ses amis. Quant à sa famille, il n'entretient pas avec elle un rapport moins subjectif. Qu'en est-il de ce rapport? «Entendre, pour nous autres, c'est normal. Mais on ne sait pas si pour lui [le Bouèbe] c'est normal ou non." Par cette réplique, Belli se montre seule capable de relativiser la surdité de son frère et, par là même, ses agissements. Aussi, cette relativisation permet de comprendre que le regard de l'enfant, parce qu'il est régi par une logique individuelle, "anime» n'importe quel objet. 


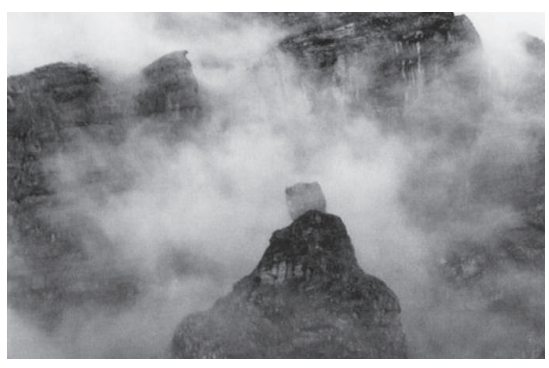

Quand il s'installe dans son refuge, le Bouèbe se met spontanément à l'ouvrage. Il vient à peine de casser la tondeuse paternelle et doit donc purger sa punition, comme son père le lui a appris, en réunissant des plaques de schiste en quantité. Mais, avant qu'il entreprenne la construction de son nouveau mur "correctionnel", une brève séquence le montre assis sur le seuil de sa nouvelle maison, en train d'observer la vallée. Brusquement, son regard s'immobilise sur un élément du paysage: un énorme rocher sur la pointe duquel un caillou est posé (fig. 12). Cet élément saillant de la montagne est, dans un premier temps, celui que le Bouèbe prend comme modèle pour fabriquer ses monticules de pierre (fig. 13). Au fur et à mesure que l'épisode évolue, le sommet de la montagne prend une signification de plus en plus intelligible. Après la visite de sa sœur et la séquence de l'inceste, le Bouèbe se retrouve seul à vivre dans le refuge et poursuit sa construction de "modèles réduits» du rocher. Un soir, alors qu'il se dirige vers son logis, il s'arrête, contemple son ouvrage achevé et regarde à nouveau la montagne. Par un raccord regard, la caméra montre en contre-plongée le rocher embrumé, duquel semble émaner un son proche d'une lamentation (fig. 14). Ce son, le Bouèbe n'est pas censé le percevoir. Pourtant, le plan qui suit cette contre-plongée représente l'enfant en larme.

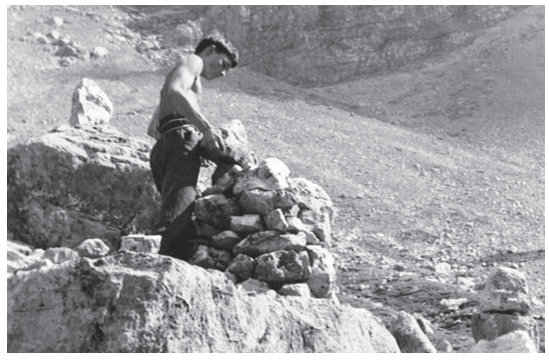




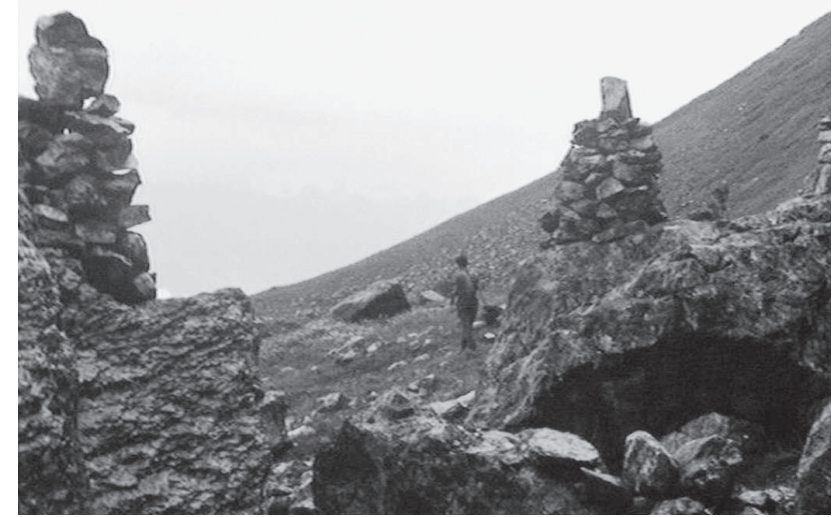

Qu'est-ce à dire sinon que cette affliction ressortit peut-être au dernier regard du Bouèbe sur sa vie d'enfant? En effet, l'épisode de sa réclusion dans le refuge a pour conséquence une double maturation: la construction initiatique des monticules de pierre et la découverte de la sexualité. Ces deux "rites» de passage à l'âge adulte sont vécus d'un côté comme une transformation corporelle - l'enfant se construit une impressionnante musculature -, d'un autre comme une transformation émotionnelle, dans la mesure où il éprouve pour la première fois de l'«amour» pour sa sœur. De plus, son évolution se donne à voir comme une élévation physique: comme si sa "renaissance» n'avait été possible que dans ces hauteurs de la montagne, dans cet univers à la fois silencieux, puissant et innocent. Ce sont là précisément trois qualités propres au Bouèbe. Tout comme lui, la montagne est sans cesse représentée comme enfant et comme adulte, à la fois libre et contrôlée, innocente et morale. La place qu'elle occupe dans Höhenfeuer semble ainsi faire d'elle un miroir, un reflet de l'enfant.

Ces quelques remarques auront peut-être contribué à mettre au jour le lien étroit qui se tisse entre le regard d'un personnage et la symbolique particulière d'un paysage. Seulement, dira-t-on, quoi de plus négativement connoté, depuis les années 1960, que d'identifier un héros à un tel lieu d'élévation? La réponse à cette question est loin d'être évidente, puisque Murer "brouille» lui-même les pistes de lecture en faisant coexister deux points de vue sur le paysage au sein du même film: celui du spectateur et celui du Bouèbe. Dans Höhenfeuer, il serait certes tentant d'apparenter l'ascension "initiatique» de l'enfant à celle de l'alpiniste dans les films de genre. Mais il faut se garder d'un pareil raccourci inter- 
prétatif: ce serait assigner au paysage représenté une dimension morale trop commune. Or, toute la consistance de ce paysage réside loin des valeurs traditionnelles et reconnaissables: elle est avant tout issue d'une réflexion profonde sur la façon dont se fait l'évolution d'un enfant, qui par sa surdité et par son lieu de naissance vit en-dehors des règles morales et des traditions imposées par son entourage, au risque de la transgression la plus violente.

$\mathrm{Au}$ lieu de plaquer sur la montagne une idéologie patriotique, Murer privilégie un regard plus individuel. Si la validité de sa symbolique peut s'affirmer dans le film, c'est justement qu'elle se fonde sur la primauté d'un regard neuf sur le monde. D'un œil enfantin, innocent, fait d'inconscience et de silence, peut se déployer un paysage alpestre nouveau, qui, vidé de toute connotation, trouve sa symbolique essentiellement en fonction d'une perception individuelle, à découvrir par le spectateur. Aussi, pour Murer, chercher ce regard singulier, ce serait avant tout ouvrir à une perception autre, plus intime, du monde qui nous entoure. 